\title{
ASSOCIAÇÃO DE CARCINOMA PAPILÍFERO DA TIREÓIDE E HIPERPARATIREOIDISMO
}

\author{
ASSOCIATION OF PAPILLARY THYROID CARCINOMA AND \\ HYPERPARATHYROIDISM
}

\author{
Fábio L. M. Montenegro, ACBC-SP'; ${ }^{1}$ Rodney B. Smith²; Inês V. Castro ${ }^{3}$; \\ Marcos R. Tavares, TCBC-SP ${ }^{4}$; Anói C. Cordeiro, ACBC-SP ${ }^{5}$; Alberto R. Ferraz, ECBC-SP ${ }^{6}$
}

\begin{abstract}
RESUMO: Objetivo: A ocorrência de carcinoma papilífero da tireóide (CPT) em doentes com hiperparatireoidismo (HPT) suscita dúvidas quanto a ser apenas coincidência ou apresentar relação causal. O objetivo deste trabalho é verificar se a incidência de CPT em diferentes formas de HPT é semelhante entre si e à incidência de CPT em achados de necropsias, assim como em doentes submetidos à tireoidectomia na mesma região. Método: Os dados de 222 pacientes consecutivos tratados por HPT foram revistos e foi analisada a incidência de CPT. Os pacientes foram estratificados em HPT primário (107) e HPT secundário (115). Os laudos anatomopatológicos foram revistos, a incidência de CPT foi pesquisada e suas características nesses indivíduos foram estudadas. Esses dados foram comparados a dados encontrados em casos de necrópsia e em 89 casos de bócio compressivo/mergulhante. Empregou-se o teste exato de Fisher e o teste t não pareado. Resultados: Os laudos foram passíveis de análise em 103 casos de HPT primário, com 10 pacientes com CPT $(9,7 \%)$ e em 111 portadores de HPT secundário, com três CPT associados $(2,7 \%)$. Houve diferença entre o HPT primário e HPT secundário $(\mathrm{p}=0,04)$. Essa diferença também foi significativa em relação aos $1 \%$ de CPT achados em necrópsia na região $(\mathrm{p}=0,0001)$. Não houve diferença com relação à incidência de $11,2 \%$ de CPT no grupo operado por compressão e também em relação às características dos tumores, apesar de haver $80 \%$ de multicentricidade no CPT de doentes com HPT primário. Conclusões: A ocorrência de CPT em HPT primário é maior que em HPT secundário e que em achados de necropsia (Rev. Col. Bras. Cir. 2005; 32(3): 115-119).
\end{abstract}

Descritores: Hiperparatireoidismo; Neoplasias da Glândula Tireóide; Tireoidectomia; Paratireoidectomia.

\section{INTRODUÇÃO}

A ocorrência de doenças da tireóide em portadores de hiperparatireoidismo (HPT) tem despertado interesse desde 1954, quando John Hellström relatou afeccções tireoidéias de interesse cirúrgico em $38 \%$ de 50 casos de HPT $^{1}$.

A observação de casos de carcinoma medular da tireóide em doentes com HPT levou à definição da entidade clínica da neoplasia endócrina múltipla (NEM) do tipo II. O que poderia parecer inicialmente uma associação fortuita de afecções de glândulas endócrinas alcançou hoje importante desenvolvimento nos aspectos diagnósticos e terapêuticos. Através da pesquisa genética em familiares de portadores da síndrome, alguns indivíduos podem ser tratados ainda antes do início do câncer na tireóide² .

A partir do relato de quatro casos de carcinoma papilífero da tireóide em doentes com HPT, a associação de neoplasia maligna diferenciada da tireóide tem gerado discussões quanto à sua incidência e mecanismo fisiopatológico nos portadores de $\mathrm{HPT}^{3}$. Essa associação varia em diferentes casuísticas entre nenhum caso até cerca de 10,7\% de carcinoma bem diferenciado da tireóide em HPT, com média de 5,39\% .
LiVolsi e Feind ${ }^{5}$ acreditavam haver incidência aumentada de câncer da tireóide sem suspeita clínica em doentes com HPT, mas não conseguiam relacionar nenhuma explicação satisfatória para o fato. Lever $\mathrm{et} \mathrm{al} .^{6}$, ainda que propensos a acreditarem numa correlação fortuita entre os dois problemas, tiveram o achado de carcinoma bem diferenciado da tireóide em 5,6\% de seus casos de HPT. Essa incidência foi diferente, com significância estatística, daquela encontrada para o câncer da tireóide achado em necropsia na sua região. Em 1994, Fedorak et al. ${ }^{7}$, com $7 \%$ de carcinoma de tireóide não medular em pacientes com HPT, confirmaram a diferença em relação ao achado de necrópsia, mencionaram que os motivos para essa associação ainda eram obscuros e que maiores esclarecimentos eram necessários. Em 1997, entretanto, Burmeister et al. ${ }^{8}$ analisaram doentes operados por HPT primário e doentes com HPT secundário/terciário e concluíram que dada a incidência semelhante de câncer da tireóide em doenças de mecanismo fisiopatológico diferentes, essa associação seria apenas uma coincidência.

Observa-se, portanto, que os dados apresentados na literatura ainda são conflitantes em relação à ocorrência de câncer bem diferenciado da tireóide em doentes com HPT. O

1. Médico Colaborador da Disciplina de Cirurgia de Cabeça e Pescoço do Hospital das Clínicas da Faculdade de Medicina da Universidade de São Paulo (HC-FMUSP).

2. Médico Colaborador da Disciplina de Cirurgia de Cabeça e Pescoço do HC-FMUSP

3. Médica da Divisão de Anatomia Patológica do HC FMUSP.

4. Professor Livre-Docente da Disciplina de Cirurgia de Cabeça e Pescoço do HC- FMUSP.

5. Ex-Professor Associado da Disciplina de Cirurgia de Cabeça e Pescoço do HC- FMUSP.

6. Professor Titular da Disciplina de Cirurgia de Cabeça e Pescoço do HC- FMUSP.

Recebido em 22/11/2004

Aceito para publicação em 02/03/2005

Trabalho realizado na Disciplina de Cirurgia de Cabeça e Pescoço, Hospital das Clínicas da Faculdade de Medicina da Universidade de São Paulo, São Paulo, Brasil. 
melhor entendimento dessa correlação, entretanto, tem interesse cirúrgico imediato. Na NEM tipo II já está bem definida a possível associação do carcinoma medular da tireóide com HPT, fato que determina investigação e tratamento dos dois problemas. Da mesma forma pode-se supor que a determinação do risco de câncer bem diferenciado da tireóide em portadores de HPT pode aumentar o cuidado nessa avaliação antes e durante a exploração cervical, com possibilidade de tratamento precoce da neoplasia maligna.

Desse modo propôs-se o estudo dessa questão no contexto brasileiro, através da análise de expressiva casuística de doentes operados por HPT primário e HPT secundário.

\section{MÉTODO}

Foram analisados retrospectivamente os pacientes operados por HPT na Disciplina de Cirurgia de Cabeça e Pescoço do Hospital das Clínicas da Faculdade de Medicina da Universidade de São Paulo de abril de 1986 a maio de 2001.

Classificou-se como HPT primário o quadro de elevação do paratormônio (PTH) às custas de uma ou mais glândulas paratireóides alteradas sem identificação de um fator metabólico antecedente desencadeante. As formas anatomopatológicas representadas no HPT primário foram: adenoma, hiperplasia primária, carcinoma, cisto, duplo adenoma ou adenoma atípico. Considerou-se HPT secundário o quadro de secreção excessiva de PTH decorrente de estimulação metabólica prévia, persistente ou não no momento da cirurgia. Dessa forma, incluíram-se sob essa denominação os doentes com insuficiência renal crônica em tratamento dialítico ou aqueles com HPT persistente após transplante renal bem sucedido. As formas anatomopatológicas corresponderam a hiperplasia de paratireóide associada ou não ao carcinoma de paratireóide.

Nos doentes operados por HPT foram analisadas as doenças concomitantes da tireóide detectadas antes ou durante a operação, demonstradas no relatório anatomopatológico. A glândula tireóide foi considerada normal quando durante a exploração cervical nenhuma área da glândula tenha se mostrado suspeita (determinando sua exérese) e quando o laudo anatomopatológico descrevia tireóide normal (em geral, nas ressecções táticas da tireóide, como margem em carcinoma de paratireóide ou na pesquisa de glândula intratireoidéia). Os laudos anatomopatológicos foram passíveis de análise em 103 casos de HPT primário e em 111 casos de HPT secundário, que representam 96,4\% dos casos operados.

Os doentes foram analisados em conjunto e divididos pelo tipo de HPT, isto é, HPT primário e HPT secundário.

Especificamente em relação ao carcinoma papilífero da tireóide foram analisadas a incidência nesses grupos e as características do tumor: tamanho, uni ou multicentricidade, invasão de cápsula, extensão extra-tireoidéia, presença de metástases linfonodais e de metástases à distância.

A incidência do carcinoma papilífero da tireóide nos doentes com HPT foi comparada a dois grupos: em casos de necrópsia e em doentes operados por aparente bócio compressivo/mergulhante.
O primeiro grupo, denominado NECRÓPSIA, foi obtido do estudo de Bisi et al. ${ }^{9}$, que analisaram a tireóide de 300 casos seqüenciais de necrópsia, na mesma região do presente trabalho. Os dados foram extraídos da publicação daqueles autores, onde consta que a idade variou entre 13 e 82 anos, com $75,8 \%$ entre a quarta e a sétima, com 100 cadáveres do sexo feminino e 200 do sexo masculino (1:2), sem evidência clínica de doença da tireóide.

O segundo grupo, designado COMPRESSÃO, foi constituído por doentes submetidos à tireoidectomia total ou parcial na DCCP-HCFMUSP, de janeiro 1998 a março 2001, por bócios nodulares com compressão de traquéia ou mergulhantes, sem suspeita pré-operatória de neoplasia, quer seja por aspectos clínicos ou citopatológicos (não foram incluídos pacientes com punção de "padrão folicular" ou punção indicativa de malignidade). No grupo COMPRESSÃO houve 89 pacientes, sendo 74 do sexo feminino e 15 do sexo masculino (4,93:1), com idade variável entre19 e 85 anos e média de 51,42 anos.

A incidência e os aspectos do carcinoma papilífero da tireóide detectados nos casos de HPT foram comparados aos achados nos grupos NECRÓPSIA e COMPRESSÃO.

A análise estatística foi realizada através de programa estatístico computadorizado GraphPad Prisma 2 (GraphPad Prism $^{\mathrm{TM}}$, Version 2.00, GraphPad Software Incorporated). As variáveis contínuas tiveram sua distribuição testada para normalidade através do método de Kolmogorov-Smirnov. Para as distribuições normais empregou-se o teste t de Student não pareado, com ou sem a correção de Welch. Para comparação entre as incidências foram utilizadas tabelas de contingência e empregou-se o teste exato de Fisher. O nível descritivo calculado foi indicado pela letra p. O nível de significância adotado foi de $5 \%$

\section{RESULTADOS}

No período do estudo houve operações por HPT em 222 pacientes, sendo 108 operações em 107 doentes com HPT primário (uma reoperação após dez anos, por recidiva de HPT, em doente submetido a paratireoidectomia total com autotransplante por NEM tipo I e que tinha uma quinta glândula paratireóide mediastinal) e 128 operações em 115 casos de HPT secundário (as reoperações foram para retirada de autotransplante, implante de paratireóide criopreservada, ressecção de quinta paratireóide mediastinal, totalização de tireoidectomia e tratamento de paratireomatose). Alguns casos representavam novas operações em doentes previamente explorados cirurgicamente em outros serviços, mas não foram incluídos como reoperações da DCCP-HCFMUSP, uma vez que foi sua primeira operação na instituição.

A idade dos pacientes variou entre seis e 83 anos (média de 45,6 anos). Os portadores de HPT primário tiveram média de idade de 48,5 anos (6 a 83 anos), com 74 indivíduos do sexo feminino e 33 do sexo masculino (2,24:1). No HPT secundário a média de idade foi de 40,1 anos (16 a 70 anos), com 64 do sexo feminino e 51 do sexo masculino $(1,26: 1)$ 
A média de idade entre os doentes com HPT primário não apresentou diferença em relação ao grupo COMPRES$\mathrm{SÃO}$ ( $\mathrm{p}=0,18$, teste $\mathrm{t}$ não pareado), mas ambos os grupos apresentaram média de idade superior à média dos casos de HPT secundário, com $\mathrm{p}<0,0001$ nas duas situações (teste t não pareado).

Quanto à distribuição pelo sexo, a diferença entre o HPT primário e HPT secundário não foi significativa $(\mathrm{p}=0,05$,

Tabela 1- Doenças da tireóide em indivíduos com HPT.

\begin{tabular}{|c|c|c|c|}
\hline Tireóide & $\begin{array}{l}\text { HPT } \\
\text { n (\%) }\end{array}$ & $\begin{array}{c}\text { HPT } \\
\text { primário } \\
\text { n }(\%)\end{array}$ & $\begin{array}{c}\text { HPT } \\
\text { secundário } \\
\text { N }(\%)\end{array}$ \\
\hline "Normal" & $122(57,0)$ & $60(58,2)$ & $62(55,9)$ \\
\hline Apenas bócio & $52(24,3)$ & $18(17,5)$ & $34(30,6)$ \\
\hline Apenas tireoidite & $14(6,5)$ & $8 \quad(7,8)$ & $6(5,4)$ \\
\hline Carcinoma papilífero & $13(6,1)$ & $10 \quad(9,7)$ & $3(2,7)$ \\
\hline Outros* & $13(6,1)$ & $7 \quad(6,8)$ & $6(5,4)$ \\
\hline Total & 214 & 103 & 111 \\
\hline
\end{tabular}

*-Bócios com tireoidite, bócio e adenoma folicular, adenoma folicular e tireoidite, fibrose, Graves.

Tabela 2 - Carcinoma papilífero da tireóide nos diversos grupos.

\begin{tabular}{lrcc}
\hline & n total & $\begin{array}{c}\text { Carcinoma } \\
\text { papilífero }\end{array}$ & $\begin{array}{c}\text { Carcinoma } \\
\text { folicular }\end{array}$ \\
\hline HPT primário & 103 & $10^{*}$ & 0 \\
HPT secundário & 111 & 3 & 0 \\
Compressão & 89 & $10^{*}$ & 3 \\
Necrópsia & 300 & 3 & 4 \\
\hline
\end{tabular}

$*_{-} p=0,81$

Tabela 3 - Aspectos dos portadores de carcinoma papilífero nos grupos.

\begin{tabular}{lrrrrr}
\hline & $\mathbf{n}$ & \multicolumn{3}{c}{ Idade } & Sexo \\
\cline { 3 - 5 } & & média & mínima máxima & \\
\hline HPT primário & 10 & 50,7 & 31 & 65 & 9F:1M \\
HPT secundário & 3 & 44,7 & 37 & 60 & $2 \mathrm{~F}: 1 \mathrm{M}$ \\
Compressão & 10 & 47,3 & 38 & 57 & $10 \mathrm{~F}: 0 \mathrm{M}$ \\
Necrópsia & 3 & - & - & - & $1 \mathrm{~F}: 2 \mathrm{M}$ \\
\hline
\end{tabular}

teste exato de Fisher). Ambos os grupos diferiram do grupo COMPRESSÃO ( $\mathrm{p}=0,03$ no HPT primário e $\mathrm{p}<0,0001$ no secundário) e do grupo NECRÓPSIA, que teve predominantemente homens.

Nos doentes operados por HPT houve achado de doenças da tireóide em $43 \%$ dos casos. Essa incidência foi semelhante nos doentes com HPT primário $(41,8 \%)$ e HPT secundário $(44,1 \%)$. A Tabela 1 mostra os achados na tireóide dos casos de HPT.

A análise das diferentes doenças nos dois tipos de HPT mostrou ocorrência significativamente maior de bócio nos doentes com HPT secundário $(\mathrm{p}=0,03)$. Entretanto a diferença não se manteve quando na análise foram incluídos os casos de bócio em associação com tireoidite $(\mathrm{p}=0,18)$.

Em relação ao carcinoma papilífero da tireóide houve incidência significativamente maior nos casos de HPT primário quando comparado ao HPT secundário $(\mathrm{p}=0,04)$. Uma doente com HPT primário havia recebido radioterapia para tratamento de tumor hipofisário prévio e teve achado de microcarcinoma papilífero multicêntrico com metástases microscópicas para linfonodos durante a cirurgia para o HPT.

O achado de carcinoma papilífero em casos de necrópsia na mesma localidade foi significativamente menor do que aquele encontrado nos doentes com HPT $(\mathrm{p}=0,0014)$. Esse resultado foi influenciado pelos casos de HPT primário ( $\mathrm{p}=0,0001)$, uma vez que o resultado observado em necrópsia $(1,0 \%)$ não diferiu daquele observado no HPT secundário $(\mathrm{p}=0,35)$.

A incidência de carcinoma papilífero da tireóide nos casos de HPT primário não apresentou diferença em relação ao achado nos pacientes tireoidectomizados por bócio compressivo, conforme mostra a Tabela 2.

Os dados dos portadores de carcinoma papilífero estão dispostos na Tabela 3. Não houve diferença significativa entre a média de idade no HPT primário e Compressão $(\mathrm{p}=0,40)$.

Em relação às características dos tumores nos diversos grupos, houve diferença na proporção de microcarcinoma nos casos de HPT primário em relação ao HPT secundário, mas sem significância estatística $(\mathrm{p}=0,56)$. As características dos tumores estão representadas na Tabela 4.

O tamanho dos tumores também não foi diferente entre o HPT primário e secundário, pela análise estatística ( $\mathrm{p}=0,26$, teste $\mathrm{t}$ não pareado com correção de Welch). O mesmo foi observado quanto à multicentricidade dos tumores ( $\mathrm{p}=0,20$ para HPT primário e secundário e $\mathrm{p}=0,16$ para HPT primário e grupo compressão). Quanto à metástase linfática também não houve diferenças significativas entre os grupos.

Tabela 4 - Características patológicas dos casos com carcinoma papilífero.

\begin{tabular}{lcccc}
\hline & Microcarcinoma & $\begin{array}{c}\text { Tamanhoem mm } \\
\text { (desvio-padrão) }\end{array}$ & Multicentricidade & $\begin{array}{c}\text { Metástase } \\
\text { Linfática }\end{array}$ \\
\hline HPT primário & $60 \%$ & $10,8 \quad( \pm 7,6)$ & $80 \%$ & $20 \%$ \\
HPT secundário & $33,3 \%$ & $28,0( \pm 18,5)$ & $33,3 \%$ & $0 \%$ \\
Compressão & $50 \%$ & $18,4( \pm 21,6)$ & $40 \%$ & $0 \%$ \\
Necrópsia & $66,6 \%$ & - & - & - \\
\hline
\end{tabular}




\section{DISCUSSÃO}

Admite-se que a primeira referência a endocrinopatias associadas tenha sido feita por Erdheim em 1903, ao descrever adenoma hipofisário e aumento de quatro paratireóides durante a necrópsia de um paciente acromegálico. Essa descrição adequa-se à de Wermer em 1954, que definiu a NEM tipo I ${ }^{10}$. Um pouco mais tarde, em 1961, Sipple notou a associação de carcinoma da tireóide em portador de feocromocitoma. Estudos subseqüentes determinaram a relação dos tumores dentro da síndrome NEM tipo II ${ }^{10}$.

O melhor entendimento dessas doenças permite planejamento do tratamento e atualmente envolve refinada análise genética. Essa análise pode permitir o diagnóstico e o tratamento do câncer da tireóide em fase precoce ou ainda antes de seu estabelecimento, com impacto significativo no controle do carcinoma medular da tireóide ${ }^{2}$.

Embora o carcinoma bem diferenciado da tireóide tenha comportamento menos agressivo que o carcinoma medular da tireóide, em geral, os resultados de seu tratamento estão ainda relacionados à completa extirpação dessas lesões neoplásicas ${ }^{11}$. Desse modo, o diagnóstico precoce desses tumores também pode representar maior chance de cura.

O reconhecimento de fatores de risco para câncer da tireóide, como irradiação cervical prévia ou exposição ambientes com contaminação de material radioativo, permite maior atenção nesses indivíduos sob risco e definição de estratégias terapêuticas ${ }^{11}$.

Há evidências de que fatores genéticos participam no desenvolvimento de doentes com carcinoma papilífero ou folicular da tireóide, como na polipose colônica familiar (síndrome de Gardner) e na Doença de Cowden. O carcinoma papilífero também tem sido observado com freqüência mais alta em algumas famílias com câncer de mama, ovário, rim e sistema nervoso central, com possível relação genética ${ }^{11}$.

Diferente do reconhecimento da alteração das paratireóides associada a carcinoma medular da tireóide na NEM tipo IIa, a ocorrência de carcinoma papilífero da tireóide em doentes com HPT ainda está mal compreendida.

Desde o relato de Ogburn e Black, em 1956³, vários autores têm descrito a associação do câncer bem diferenciado da tireóide em HPT. Para alguns a relação é puramente casual ${ }^{8}$, para outros possivelmente casual, mas com maior incidência do que o esperado ${ }^{6,7,12}$ e muito provavelmente não casual para outros ${ }^{5,13-15}$.

Em alguns casos observou-se que as duas doenças ocorreram após irradiação cervical ${ }^{16,17}$. Embora possa ser fator etiológico importante, como a possível causa num dos casos desta casuística, a irradiação prévia é identificada em cerca de $30 \%$ dos casos de carcinoma de tireóide e HPT $^{18}$. Assim, a exposição à radiação não pode ser a explicação para todos os casos, mas deve ser investigada antes da exploração cervical por HPT.

No presente estudo houve diferença significativa entre a incidência de carcinoma papilífero da tireóide em doen- tes com HPT primário e HPT secundário. Essa observação difere da de Burmeister et al.$^{8}$, que, ao determinarem incidência semelhante entre doentes com HPT primário e HPT secundário, consideraram a associação puramente fortuita. Burmeister et $a l .{ }^{8}$ afastaram a relação de causalidade do carcinoma papilífero da tireóide por notar sua igual ocorrência em doentes com HPT cujo mecanismo fisiopatológico é diverso.

A comparação com dados de necrópsia da mesma região mostrou significativa proporção de doentes com câncer da tireóide e HPT primário. A grande diferença entre o sexo nos diferentes grupos talvez possa ter afetado o resultado. Isso justifica novo esforço para análise em casos de necrópsia em mulheres cuja faixa etária aproxime-se dos casos aqui apresentados.

A comparação com doentes submetidos a tireoidectomia por doença multinodular sem suspeita inicial de câncer parece inédita até onde os autores puderam obter dados da literatura. Embora criticável por também representar um grupo de doentes com maior risco para presença de câncer de tireóide, ela representa um grupo que está tendo maior atenção para a tireóide pela equipe médica, de forma similar aos doentes tratados por HPT. A semelhança de comportamento nos dois grupos (HPT primário e grupo compressão) favorece a hipótese de associação casual entre o câncer e o HPT.

A análise das características dos tumores chama atenção para a maior freqüência de microcarcinomas em HPT primário do que no HPT secundário, mas não houve demonstração dessa diferença, que pode ser apenas aparente. Ainda assim, vale observar a multicentricidade expressiva e a possibilidade de metástases cervicais. Essas observações fortalecem o emprego da tireoidectomia total como menor operação para o adequado manejo desses pacientes, que contrasta com a opinião de Linos et al..$^{18}$.

O presente estudo não pode assegurar relação causal entre os dois problemas, embora tenha mostrado alta incidência de carcinoma papilífero da tireóide em portadores de HPT primário no nosso meio. A casuística de HPT ora analisada é uma das maiores no Brasil, fruto do trabalho do Prof. Dr. Anísio Costa Toledo e de seus seguidores na DCCP-HCFMUSP, por ele fundada. Não foi observada nenhuma menção ao problema do câncer da tireóide em HPT em nosso meio, mesmo em outras séries muito significativas de HPT primário ${ }^{19}$. Parece provável que outros casos tenham sido tratados por cirurgiões brasileiros, mas os dados não estão disponíveis. A criação de um banco de dados brasileiro de doentes tratados por HPT seria útil para aprofundar esse e outros questionamentos relacionados às doenças das paratireóides e esforços devem ser envidados junto aos órgãos de saúde para essa realização.

Se realmente houver causalidade na relação do carcinoma papilífero da tireóide e HPT, o tratamento precoce do problema poderá ajudar vários doentes. Mais ainda, definindo-se melhor o mecanismo fisiopatológico ou fatores de risco para essa associação, talvez seja possível o delineamento de estratégias para prevenção. 


\begin{abstract}
Background: Association of papillary thyroid carcinoma (PTC) and hyperparathyroidism (HPT) has not been clearly defined. The incidence of PTC in different types of HPT and necropsy or patients submitted to thyroidectomy in the same region is analyzed to verify if this association is casual or not. Methods: Data of 222 consecutive patients operated for HPT were reviewed and incidence of PTC was defined. Patients were analyzed as primary HPT(107) and secondary (115). The incidence of PTC was compared to that found in necropsy and to that observed in 89 patients with compressive multinodular goiter submitted to thyroidectomy. Statistical analysis included Fisher's exact test and Student's test. Results: Pathology reports were available in 103 cases of primary HPT, and in 10 patients PTC was detected (9.7\%). In 111 secondary HPT patients, PTC was found in three $(2.7 \%)$, with statistical significant difference between primary and secondary HPT $(p=0.04)$. This difference was also significant of the $1 \%$ incidence of PTC found in necropsy in the same area $(p=0.0001)$. No difference was observed in relation to the incidence of $11.2 \%$ of PTC found in patients operated for compressive goiter. Tumor characteristics were not statistically different, although multicentricity was detected in $80 \%$ of PTC of patients with primary HPT. Conclusions: The incidence of PTC was elevated in patients with primary HPT, in relation to necropsy or secondary HPT cases.
\end{abstract}

Key-words: Hyperparathyroidism; Thyroid gland neoplasms; Thyroidectomy; Parathyroidectomy.

\section{REFERÊNCIAS}

1. Hellström J. Primary hyperparathyroidism: observations in a series of 50 cases. Acta Endocrinol. 1954;16:30-58.

2. Wells SA, Chi DD, Toshima K, et al. Predictive DNA testing and prophylactic thyroidectomy in patients at risk for multiple endocrine neoplasia type 2A. Ann Surg. 1994;220(3):237-42.

3. Ogburn PL, Black BM. Primary hyperparathyroidism and papillary adenocarcinoma of the thyroid: report of four cases. Proc Staff Meet Mayo Clin. 1956;31:295-8.

4. Regal M, Páramo C, Luna Cano R, et al. Coexistence of primary hyperparathyroidism and thyroid disease. J Endocrinol Invest. 1999;22(3):191-7.

5. Li Volsi VA, Feind CR. Parathyroid adenoma and nonmedullary thyroid carcinoma. Cancer. 1976;38(3):1391-3

6. Lever EG, Refetoff S, Strauss FH, et al. Coexisting thyroid and parathyroid disease-are they related? Surgery. 1983;94(6):893900.

7. Fedorak IJ, Salti G, Fulton N, et al. Increased incidence of thyroid cancer in patients with primary hyperparathyroidism: a continuing dilemma. Am Surg. 1994;60(6):427-31.

8. Burmeister LA, Sandberg M, Carty SE, et al. Thyroid carcinoma found at parathyroidectomy: association with primary, secondary, and tertiary hyperparathyroidism. Cancer. 1997;79(8):1611-6.

9. Bisi H, Fernandes VS, Camargo RY, et al. The prevalence of unsuspected thyroid pathology in 300 sequential autopsies, with special reference to the incidental carcinoma. Cancer 1989;64(9):1888-93.

10. Wells SA Jr, Llairmore TC. The multiple endocrine neoplasias. In: Sabiston DC Jr. Textbook of surgery. 14 ed. Philadelphia. WB Saunders. 1991. p.590-597.

11. Gagel RF, Goepfert H, Callender D. Changing concepts in the pathogenesis and management of thyroid carcinoma. CA Cancer J Clin. 1996;46(5):261-83
12. Kairaluoma MI, Heikkinen E, Mokka R, et al. Non-medullary thyroid carcinoma in patients with parathyroid adenoma. Acta Chir Scand. 1976;142(6):447-9.

13. Ellenberg AH, Goldman L, Gordon GS et al. Thyroid carcinoma in patients with hyperparathyroidism. Surgery. 1962;51:708 17 apud Nishiyama RH, Farhi D, Thompson NW. Radiation exposure and the simultaneous occurrence of primary hyperparathyroidism and thyroid nodules. Surg Clin North Am. 1979;59(1):65-75.

14. Newman HK, Plucinski TE. Unsuspected nonmedullary carcinoma of the thyroid in patients with hyperparathyroidism. Am J Surg. 1977;134(6):799-802.

15. Calcaterra TC, Paglia D. The coexistence of parathyroid adenoma and thyroid carcinoma. Laryngoscope. 1979;89 (7 Pt 1):1166-9.

16. Nishiyama RH, Farhi D, Thompson N. Radiation exposure and the simultaneous occurrence of primary hyperparathyroidism and thyroid nodules. Surg Clin North Am. 1979;59(1):65-75.

17. De Jong SA, Demeter JG, Jarosz BA, et al. Thyroid carcinoma and hyperparathyroidism after radiation therapy for adolescent acne vulgaris. Surgery. 1991;110(4): 691-5.

18. Linos DA, van Heerden JA, Edis AJ. Primary hyperparathyroidism and nonmedullary thyroid cancer. Am J Surg. 1982;143(3):301-3.

19. Santos RO. Hiperparatireoidismo primário: aspectos clínicos, laboratoriais, cirúrgicos e histopatológicos em 48 pacientes. Dissertação (Mestrado). São Paulo. Universidade Federal de São Paulo. 2000. 138p.

Endereço para correspondência:

Rua Apeninos, 1118 apto 62

Paraíso São Paulo SP

CEP 04104-021

E-mail: fabiomonte@uol.com.br 